\title{
Legitimation oder Auslegung? \\ Die römischrechtlichen Quellenverweise vom Baltischen Privatrecht in den Riga'schen Stadtgerichtsentscheidungen
}

\begin{abstract}
This article deals with the goal of the references to Roman law in the Baltic Private Law Act (BPLA), a law, which did enter into force in 1865. The BPLA was the collection of laws that was in force so far and as such it had to have under articles references to the sources of the articles. The author of present article has so far analysed the compatibility of the references to Roman law with the article text and the origin of article texts. On the bases of the analyses the main purpose of BPLA so far identified was to legitimise the code of laws both for Russian central authorities and local lawyers. In present article the use of the references to Roman law in the decisions of the city courts of Riga is examined. The examination showed that referred Roman law was used quite often also for the interpretation of the articles and so Roman law was had double purpose: to legitimate the code, but also for the interpretation.
\end{abstract}

\section{Einleitung}

Im Jahre 1865 trat in den drei Ostseeprovinzen des Russischen Imperiums - Estland, Livland und Curland ${ }^{1}$ ein neues Gesetzbuch, das LECP in Kraft. Bis zu diesem Zeitpunkt hatten die drei Provinzen nach den Unterwerfungskapitulationen von 1710/1795 im russischen Imperium eine rechtliche Sonderstellung genossen und während der 18-19. Jahrhundert die hergebrachte Rechtsordnung beibehalten. ${ }^{2}$

Das LECP sollte eine Kodifikation - eigentlich Kompilation - der ostseeprovinziellen Rechte sein, in die das gesamte geltende Privatrecht zusammengesammelt werden musste. Das Inkrafttreten dieser „Kodifikation“ bedeutete eine entscheidende Zäsur für die Rechtsquellenlage. Vor der Kodifikation galten nebeneinander die unterschiedlichsten Rechtsmassen: von verschiedenen Geschichtsperioden und Herrschaf-

\footnotetext{
${ }^{1}$ Diese Provinzen umfassen das Territorium der heutigen Staaten Estland und Lettland.

2 Zu den Kapitulationen: LuTs, Modernisierung $159 \mathrm{f}$.
}

ten stammende Stadt- und Landrechte, Landesund (im Baltikum verschiedene) Reichsrechte, Privilegien, Polizeiordnungen, Gewohnheitsrecht, und nicht zuletzt, obwohl subsidiär, das sog. gemeine Recht als eine Mischung des römischen, kanonischen und ggf. Reichsrechts des Heiligen Römischen Reichs.

Mit dem Inkrafttreten der Kodifikation sollte das Recht nunmehr von einem einzigen Gesetzbuch stammen, das sowohl Vollständigkeit als auch System anstrebte. ${ }^{3}$

\section{Die römischrechtlichen Quellenverweise im LECP}

Das LECP sollte nach Vorgaben des Zaren die "Codification des bestehenden Rechts" sein. Daher war vorgesehen, dass bei jedem Artikel

\footnotetext{
${ }^{3}$ Zur Kodifikationsgeschichte und Begriffe: CARONI, Gesetz. Zum LECP als Kodifikation: SIIMETS-GROSs, Einzige Quelle 276.
} 
auf die Quellen verwiesen werden musste. ${ }^{4}$ Im Folgenden wird ein Beispiel für diese Gesetztechnik gebracht:

,1091. Das Bestehen einer Dienstbarkeit wird nie vermuthet, muss vielmehr im Zweifel von dem Berechtigten bewiesen werden.

L. 9. L 14 D. de servitut. praed. urban. (VIII, 2). L. 16 D. de servitut. praed. rust. (VIII, 3). L. 4. 6. 8. 9. 11 C. de servitut. (III 34)."

Wie das Beispiel zeigt, gab es unter den Artikeln meistens nicht wenige Quellenverweise und viele davon waren auf das römische Recht bezogen. Die Quellenverweise wurden außerdem im Quellenregister zum Gesetzbuch angeführt. Aufgrund dieses Registers ist der Anteil der Verweise auf die verschiedenen Quellen errechnet worden: beim römischen Recht war es der höchste mit 57,2\% von insgesamt etwa 4600 Artikeln des LECP (vor allen einheimischen Rechtsquellen mit $40 \%) .{ }^{5}$ Deswegen wurde die Kompilation noch 1918, als sie immer noch in Kraft war, als Triumph des römischen Rechts gelobt, da es „nicht nur in dem Umfang übernommen [war], in welchem es bisher als Subsidiarrecht gegolten hat, sondern das ganze Werk der Kodifikation [wurde] auf streng römisch-rechtlicher Grundlage aufgebaut. [...]“6

In der Tat handelte es sich statt eines Triumphes des römischen Rechts um einen Triumph der römischrechtlichen Quellenverweise, die es im Übermaß gab, die aber oft falsch waren.7 Insgesamt gibt es im Gesetzbuch eher zu viel als zu wenig Quellenverweise. Es wurden möglichst viele Referenzen hinzugefügt, die sich auch öfters wiederholten, teilweise nicht sachgemäß waren oder als bloße Verzierung wirken. Es gab

\footnotetext{
${ }^{4}$ Greiffenhagen, Bunge 25. Zu den Kompilationsgrundsätzen des Baltischen Privatrechts: LUTS, Private Law 157-167.

${ }^{5}$ LUTS-SOOTAK, Das Baltische Privatrecht 369.

${ }^{6}$ YlANDER, Die Rolle 441.

${ }^{7}$ Dazu SiIMETS-Gross, Roman Law Origin 313-330; SIIMETS-Gross, Römisches Recht im Baltikum 83-93.
}

außerdem eine Fülle von fehlschlagenden Quellenverweisen. Wenn man aber berücksichtigt, dass in der Regel wenigstens eine Referenz von mehreren der bei einem Artikel angegebenen sachgemäß war, kann daraus geschlossen werden, dass die Quellenverweise auch nicht ganz willkürlich hinzugefügt worden waren. ${ }^{8}$

\section{Die möglichen Funktionen der Quellenverweise im Baltischen Privatrechtsgesetzbuch}

Ein Grund, warum man beim LECP vom Triumph des römischen Rechts spricht, ist dieser Reichtum der Verweise auf die römischen Quellen. Bei einer näheren Analyse gibt es für einen Triumph aber zu viele unrichtige oder ungenaue Referenzen. Daher stellt sich die Frage, welche Rolle oder Funktionen die Quellenverweise hatten.

Die erste und formelle Funktion war es, zu zeigen, dass die Vorgabe des Zaren, bei den Gesetzesartikeln auf deren Quellen zu verweisen, erfüllt worden war. Das Erfordernis kam aus den allgemeinen Grundsätzen der zeitgenössischen zarenrussischen Kodifizierungsideologie und sollte zeigen, dass das LECP eine Sammlung der geltenden Rechte ist. Das Fehlen von Verweisen hätte daher bedeutet, dass es sich um neues Recht gehandelt hätte, das nicht in die Kompilation gehörte. Die Quellenverweise sollten zeigen, dass die Vorgabe des Zaren eingehalten und der konservativen Ideologie gefolgt wurde. Das Quellenregister sollte dies nochmals betonen. ${ }^{9}$

Vor allem wäre aber logisch, dass die Quellenverweise künftig bei der Auslegung der Quellentexte benutzt werden könnten. Dies wurde z.B. von Aage Ylander aus Berlin, zu dessen

\footnotetext{
8 SIIMETS-GROSS, Römisches Recht im Baltikum 93-95.

${ }_{9}^{9}$ SiIMETS-Gross, Römisches Recht im Baltikum 111.
} 
Person keine näheren Angaben bekannt sind, obwohl er dem Namen nach aus dem Baltikum stammen könnte, behauptet und folgendes Auslegungsprinzip beschrieben: „Bei der Auslegung wird man sich strikt an die in den Fußnoten angegebenen Quellen halten müssen, welche, wie schon gesagt, in der überwiegenden Mehrzahl von Fällen dem römischen Recht angehören. Natürlich hat aber der Gesetzestext immer dem Quellentext vorzugehen [...]. ${ }^{\prime 10}$

In diesem Fall wäre das Quellenregister nicht nur als Rechtfertigung der konservativen Ideologie nötig gewesen, sondern auch, um die Suche der für die exaktere Auslegung nötigen Quellen zu erleichtern. Ylander sagte jedoch nicht, ob nach dem Inkrafttreten des LECP die Benutzung der Quellentexte zur Auslegung auch in der Praxis stattgefunden hat. ${ }^{11}$

Marju Luts-Sootak, Professorin für Rechtsgeschichte in Tartu, hat die das Provinzialrecht betreffenden Entscheidungen des russischen obersten Gerichtshofs, des dirigierenden Senats ${ }^{12}$, untersucht. Es handelte sich um die Entscheidungen des Senats zu den estländischen Rechtsstreitigkeiten in den 1880er Jahren. In dieser Zeit kamen die Entscheidungen, die nach LECP getroffen wurden, bei diesem Senat an. Von den analysierten Fällen haben sich 36 mit prozeßrechtlichen, 16 u.a mit materiellrechtlichen Fragen beschäftigt. Von 52 Entscheidungen gab nur zwei, in denen in der Argumentation römischrechtliche Verweise des LECP genannt oder benutzt waren. Damit war die Benutzung der Verweise eher Ausnahme als Regel. ${ }^{13}$

Der Professor für Provinzialrecht an der Universität zu Dorpat im 19. Jahrhundert, Carl Eduard

\footnotetext{
10 YLANDER, Die Rolle 445.

11 SIIMETS-GROSS, Römisches Recht im Baltikum 111.

12 Der Dirigierende Senat war sowohl der höchste Gerichtshof als auch die hohe Verwaltungsbehörde des russischen Reichs.

${ }^{13}$ LUTS-SOOTAK, Russische Reichsjustiz 294.
}

Erdmann, ${ }^{14}$ war aber der Meinung, dass die Quellentexte nur zum Nachschlagen ähnlicher Gebiete angewandt werden könnten:

„Vielmehr werden diese citirten Quellen häufig als bloße Angaben der entsprechenden Gebiete anderer Rechte zum Zwecke des Nachschlagens zu verwenden sein." 15

Nach seiner Ansicht verweist die Kodifikation zwar auf Quellen, „ohne jedoch den citirten Quellenstellen dadurch einen ausschließlichen oder gar gesetzesgleichen Character geben zu wollen" ${ }^{16}$ Daher hätten die Quellentexte keine Gesetzeskraft und sollten auch nicht wie Quellen des bisher geltenden Rechts zur Auslegung unbedingt nötig sein. Seine Ansicht schloss aber nicht aus, dass Erdmann selbst die verwiesenen Quellen benutzte, um die erwünschte Auslegung des Artikeltextes zu erreichen. Z.B. enthält Art. 529 des LECP eine von den römischen Quellen abweichende Definition der körperlichen und unkörperlichen Sachen. Bei der Interpretation dieses Artikels in seinem vierbändigen Werk „System des Privatrechts der Ostseeprovinzen Liv-, Est- und Kurland" benutzte Erdmann die Quellenreferenzen zusammen mit anderen Auslegungsmethoden, um zu dem nach seiner Ansicht richtigen Ergebnis zu kommen. ${ }^{17}$ Bisher ist dies zwar die einzige entdeckte Stelle, in der er die Quellenverweise zur Auslegung in dieser Weise benutzte, aber anscheinend war

\footnotetext{
${ }^{14}$ Erdmann (1841-1898) begann 1858 an der Universität Dorpat das Studium der Philosophie, 1859-1862 studierte er Rechtswissenschaft in Dorpat und Heidelberg und erwarb den Grad des Kandidaten. 18641869 war er Sekretärgehilfe und Ratssekretär in Mitau, 1869-1872 Universitätssyndikus in Dorpat. 1870 wurde er Mag. iur. in Dorpat. 1870-1872 war er Privatdozent in Dorpat, 1872 erwarb er den Grad Dr. iur. in Dorpat, 1872 wurde er außerordentlicher, 1873 ordentlicher Professor. 1893 wurde er im Zuge der Russifizierung der Universität entlassen und lebte bis 1898 im Ruhestand.

${ }^{15}$ ERdMANN, System 35, Anm. 3.

${ }^{16}$ ERDMANN, System 35.

17 ERDMANN, System 134.
} 
diese Vorgehensweise für ihn nicht ausgeschlossen. Dabei betonte Ylander in der oben zitierten Passage, dass der Text der Quelle, auf die bei einem Artikel verwiesen wurde, keinen Vorzug vor dem Text des Artikels haben konnte. ${ }^{18}$ Wenigstens ausnahmsweise wurde dies aber doch gemacht. Die drei genannten Fälle, in denen die Quellenverweise zur Auslegung benutzt wurden, zeigen, dass die Auslegung zwar möglich war, aber selten vorgenommen wurde.

Nur aufgrund der Untersuchung der Praxis des höchsten Gerichts, des russischen dirigierenden Senats könnte behauptet werden, dass die Quellenverweise zur Auslegung kaum benutzt wurden. Daher könnte die Hypothese aufgestellt werden, dass die Quellenverweise hauptsächlich zur Legitimation des Gesetzbuches, dessen Inhalts und der anderen Rechtsquellen wie das Gewohnheitsrecht gedacht waren.

Eine solche Rechtfertigung oder Legitimierung könnte der Verfasser des LECP Entwurfs, Friedrich Georg von Bunge ${ }^{19}$ bei den Praktikern der Ostseeprovinzen als nötig angesehen haben. Da diese bisher das römische Recht viel benutzt hatten $^{20}$, ist es verständlich, dass sie das auch gerne fortgesetzt hätten. So hätte die angeblich rege Benutzung des römischen Rechts, das daher ebenfalls weiterhin benutzt werden konnte, Bunges Unternehmen bei den einheimischen

\footnotetext{
18 YLANDER, Die Rolle 445.

${ }^{19}$ Bunge (1802-1897) studierte in Dorpat von 18181822 Kameralistik und Rechtswissenschaft. 1823 erwarb er den Kandidatengrad (d.h. einen Universitätsabschluss mit mündlicher Prüfung und einer wissenschaftlicher Abhandlung). Seit 1825 war er Privatdozent der Provinzialrechte, daneben 1825-1830 Stadtsyndikus und Ratsherr von Dorpat. 1827 erwarb Bunge den Doktorgrad der Universität Heidelberg in absentia. 1831-1842 war er außerordentlicher, danach ordentlicher Professor der Provinzialrechte in Dorpat. 1843-1856 war Bunge Stadtsyndikus und Ratsherr in Reval. 1856-1865 war er Beamter der Kodifizierungsabteilung in St. Petersburg. 1869-1897 lebte er im Ruhestand in Gotha und Wiesbaden.

${ }^{20}$ So z.B MADAI, Rezension 845.
}

Praktikern legitimiert oder die allgemeinmenschliche Scheu vor dem Umlernen minimiert.

Auch das provinzielle Privatgesetzbuch als solches konnte eine Legitimation gebrauchen. Während des jahrzehntelang dauernden Kodifizierungsprozesses hatte sich die allgemeine russische Kodifikationsideologie und rechtspolitische Grundhaltung geändert. Der ursprünglichen Absicht, nur das geltende Recht (und damit auch alle regionalen Einzelheiten) zu konsolidieren, wurde das Ziel, mit der Kodifikation die Gesetzgebung des russischen Imperiums zu vereinheitlichen, entgegengesetzt. Sonderregelungen der Provinzen wurden generell nicht mehr toleriert. ${ }^{21}$ Ein einheimisch geprägtes Provinzialgesetzbuch für die Ostseeprovinzen wäre mit diesem Ziel eigentlich nicht vereinbar gewesen.

Darüber hinaus herrschte in den reform- und unifizierungsgesinnten Regierungskreisen Russlands in den 1860er Jahren unter dem Reformzaren Alexander II. eine unleugbare RomBegeisterung. ${ }^{22}$ Ein Gesetzbuch, das den Anschein erweckte, es beruhe auf römischrechtlichen Grundsätzen, konnte leichter akzeptiert werden als ein Gesetzbuch, in dem hauptsächlich die baltischen Sonderrechte als Quellen vorkamen. So ist es möglich, dass die vielen römischen Quellenverweise unter anderem zur Beruhigung der russischen Zentralmacht dienen sollten. ${ }^{23}$

\footnotetext{
${ }^{21}$ Näher zur Situation in Russland: BABEROWSKI, Justizwesen 156f.

22 Näher: SIIMETS-GROSS, Roman Law Triumph $188 \mathrm{f}$.

${ }^{23}$ Ebd. 188-189.
} 


\section{Die Benutzung der römisch- rechtlichen Quellenverweise in den Stadtgerichtsentscheidungen von Riga}

\subsection{Gegenstand der Analyse}

Die Benutzung der Quellenverweise in der Argumentation der provinziellen Gerichte ist bisher noch nicht untersucht worden. Einige Jahre nach dem Inkrafttreten LECP hat Victor Zwingmann, Assessor des Riga'schen Landvogteigerichts eine Sammlung "Civilrechtliche Entscheidungen der Riga'schen Stadtgerichte" (Riga 1871) herausgegeben. Die vorliegende Untersuchung wurde aufgrund dieser Edition durchgeführt, aus der die sachenrechtlichen Entscheidungen ausgewählt wurden.

Im ersten Band der „civilrechtlichen Entscheidungen“, der 171 Urteile von 1865 bis 1870 enthält, gibt es 32 sachenrechtliche Entscheidungen von 171, wobei einige sachenrechtliche Entscheidungen eigentlich handelsrechtliche Fragen betrafen. Unter den Urteilen nehmen die sachenrechtlichen nach den obligationenrechtlichen und vor den familien- und erbrechtlichen den zweiten Platz ein. Jedes Urteil hat die Hauptfrage der Entscheidung zum Titel, der Herausgeber hat aber sowohl die Argumentationen des Klägers und des Beklagten als auch die Entscheidungsgründe des Gerichts gekürzt.

Statistisch kann gesagt werden, dass der Anteil der römisch-rechtlichen Verweise in den Entscheidungen der Riga'schen Stadtgerichte viel grösser war als bisher anhand der Senatsentscheidungen angenommen werden konnte. In elf von 32 Entscheidungen gab es römischrechtlichen Verweise (21 waren also ohne); dazu kommen noch fünf weitere, in denen das römische Recht erwähnt worden ist oder nur auf die Pandektenlehrbücher verwiesen worden ist, ohne Quellen zu erwähnen. Auch wenn das gemeine Recht und damit Pandektenlehrbücher nicht mehr zum geltenden Recht gehörten, wur- de oft in den Entscheidungen, aber auch von Parteien auf Pandektenlehrbücher als Grundlage der Argumentation verwiesen. Zusammenfassend kann man die Benutzung in der Argumentation folgendermaßen klassifizieren: der Kläger streicht die römischrechtlichen Quellen als Stütze seiner Argumentation heraus, aber das Gericht wendet sie nicht an; die römischrechtlichen Quellen werden zwar genannt, aber deren Relevanz für die Entscheidung wird verneint; die römischrechtlichen Quellen bestätigen die Ansicht des Gerichts; die Geltung des römischen Rechts wird bejaht, ohne auf die Quellen hinzuweisen. Die folgende Analyse folgt dieser Einteilung.

\subsection{Die römisch-rechtliche Quellen in der Argumentation des Klägers}

Im Text der Entscheidungen wird manchmal auch die Argumentation der Parteien gebracht. Einmal wurde in den sachenrechtlichen Entscheidungen vom Kläger zur Bedeutung von fundus auf D 50, 16, 211 verwiesen (von der Beklagtenseite wurden keine Verweise auf das römische Recht gebracht). Das Gericht hat befunden, dass „die klägerischerseits allegirte 1. 211 Dig. De V.S. zu demselben Resultate führt, indem sie besagt, daß der Ausdruck Grundstück (fundus) sowohl die Grundfläche (ager) als auch die darauf befindlichen Gebäude umfassen [...].“24 Trotzdem hat das Gericht anders als vom Kläger gewollt entschieden.

\subsection{Relevanz der römisch-rechtlichen Quellen für die Entscheidung wird verneint}

Im Prinzip wäre möglich, dass die römischrechtliche Verweise zwar vorhanden sind, aber ihre Anwendbarkeit verneint wird, dies ist aber

${ }^{24}$ Nr. 23a. und b. Rechtskräftiges Erkenntniß der I. Section des Landvogteigerichts vom 6. Nov. 1870, No. 206, in: ZWINGMANN, Civilrechtliche 43. 
nur in drei Fällen so (Urteile Nr. 29, 39, 50). In zwei von diesen (29 u. 50) hat das Gericht der ersten Instanz auf die römischrechtlichen Quellen verwiesen und das Gericht der zweiten Instanz die Anwendbarkeit verneint oder gefunden, dass diese Quellen und Artikel nicht das Problem lösen.

Als Beispiel sei auf das Urteil 50 verwiesen, wo es um die Frage nach der Befriedigungsreihenfolge der Pfandrechte geht - und eigentlich um eine Frage nach dem Riga'schen Stadtrecht. Trotzdem ist das römische Recht auch hier relevant, da u.a der Inhalt des entsprechenden Artikels von LECP analysiert wurde. Der Fall wurde in der Appellationsinstanz endgültig gelöst und das Gericht hat befunden:

„Iudex a quo hat seine Entscheidung auf die 1. 2 Dig. XX, 4 gestützt [...] und sieht eine Bestätigung dieses Grundsatzes auch in den Stadtrechten Lib. III Tit. $X$ pct. 12 und 13, sowie in dem Art. 1612 des 3. Bandes des Prov. Rechts [d.h. LECP].“25 Dann fand das Appellationsgericht aber, dass „der Grundsatz, daß das ältere generelle Pfandrecht das jüngere specielle Pfandrecht ausschließe, ist demnach in den angezogenen Bestimmungen der Stadtrechte und des Provinzialrechts nicht vorhanden und überhaupt von dem provinziellen Recht nie anerkannt worden.“26 Stattdessen komme der Grundsatz, dass das spezielle Pfandrecht dem generellen vorgeht, aus dem Gewohnheitsrecht, und sei durch Praxis und Doktrin anerkannt worden. ${ }^{27}$

In diesem Fall (und auch in Fall 29) hat das erstinstanzliche Gericht gefunden, dass das Problem

\footnotetext{
${ }^{25}$ Nr. 50. Appellationserkenntniß der II Instanz [d.h Landvogteigericht] vom 10. Sept. 1869, No.1865, in: ZWINGMANN, Civilrechtliche 102.

${ }^{26}$ Nr. 50. Appellationserkenntniß der II Instanz [d.h Landvogteigericht] vom 10. Sept. 1869, No.1865, in: ZWINGMANN, Civilrechtliche 102.

${ }^{27}$ Nr. 50. Appellationserkenntniß der II Instanz [d.h Landvogteigericht] vom 10. Sept. 1869, No. 1865, in: ZWINGMANN, Civilrechtliche 102-103.
}

durch die Normen mit römischrechtlichen Grundlage zu entscheiden ist. Die Meinung wird von der zweiten Instanz aber verworfen und die Geltung dieser Normen daher verneint.

\subsection{Die römischrechtlichen Quellen bestätigen die Ansicht des Gerichts}

Meistens wird die Stelle aus dem römischen Recht zur Bestätigung der Ansicht des Gerichts benutzt (Urteile 24, 37, 41, 46, 49), oder auch zur bestätigenden Auslegung: so ist es z.B. in einer Entscheidung „über das Rechtsverhältnis an Grenzzäunen in den Städten“28. In dieser Entscheidung geht es um die Problematik, ob und in welchen Fällen Grenzzäune der Grundstücke in den Städten im Alleineigentum, im Gemeinschaftseigentum oder Miteigentum sind. Nach einer längeren Erörterung sagt das Gericht, dass - auch wenn der Nachbar Miteigentum am

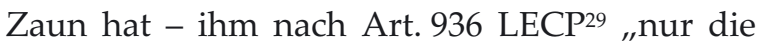
Benutzung der seinem Grundstücke zugewandten Seite und auch dies mit der Einschränkung gestattet, daß dadurch keine wesentliche Veränderung der Mauer herbeigeführt werde, wobei die als Belegstelle allegirte $1.19 \S 1$ D. VIII, 2 noch speziell bestimmt, daß ein Gewölbe an die gemeinsame Mauer nur der Art gelegt werden darf, daß dasselbe auch ohne die Mauer ruhen kann, welche Vorschrift auch auf den gemeinschaftlichen Zaun analoge Anwendung finden muß."30 Der römischrechtliche Verweis

\footnotetext{
${ }^{28}$ Nr. 31. Rechtskräftiges Erkenntniß der I. Section des Landvogteigerichts vom 8. Juli 1869, No.206, in: ZWINGMANN, Civilrechtliche 59-62.

${ }^{29}$ Es steht im 5. Hauptstück des LECP unter dem Teil "Von dem Miteigenthum". Die Verweise unter den Artikel waren D 8, 2, 8; D 8, 2, 13, pr-1; D 8, 2, 19; D 9, 2, 27, 10 und auf Lübisches Stadtrecht, Buch 3, Tit. 12, Art. 5. Alle römisch-rechtliche Verweise sind unter diesem Artikel relevant.

${ }^{30}$ Hervorhebung durch die Autorin. Nr. 30. Rechtskräftiges Erkenntniß der I. Section des Landvogteigerichts vom 8. Juli 1869, No. 206, in: ZwINGMANN, Civilrechtliche 62 .
} 
zu Art. 936 LECP wurde auf eine bestätigende Weise benutzt, um den Inhalt des Artikels zu erläutern und durch Analogie zu erweitern. Hier kann gesagt werden, dass durch den Quellenverweis auch praktischer Nutzen gewonnen werden konnte und er daher nicht nur als „Verzierung" diente.

Davon unterscheidet sich das nächste Beispiel (Urteil 32), wo die Digestenstelle eher als „Verzierung" benutzt worden ist (so auch teil $41^{31}$ ). Es handelt sich um die Frage, ob ein an dem Zinsgrundstück begrenzende Gewässer dem Grundzinsherren oder Grundzinsberechtigten gehört. ${ }^{32}$ Das Gericht war der Ansicht, dass der Grundzinsberechtigte das Grundstück nur in den Grenzen des Grundstücks benutzen darf. Der Streit betrifft die Art. 947 und 1325 LECP, die auch römischrechtliche und andere Verweise anthalten, aber der Riga'sche Rat hat sich für eine andere Digestenstelle als die unter den Artikeln genannten entschieden. Das Gericht sagt: „Dabei kann es keinen Unterschied machen, ob dieser Theil Land oder Wasser ist, wie denn auch schon das römische Recht ausdrücklich ausspricht, daß ein Privatfluß sich von anderen Privatplätzen in Nichts unterscheide (1.4 Dig. 47, 12). "33 Dabei hat das Gericht die Stelle offenbar aus dem Gedächtnis zitiert, da in dieser Stelle tatsächlich etwas völlig anderes steht: "Sepulchra hostium religiosa nobis non sunt: ideoque lapides inde sublatos in quemlibet usum convertere possumus: non sepulchri violati actio competit." Das heißt, dass die Grabstellen der Feinde für uns keine religiösen Stellen sind; daher können wir die Steine, die aus denen weggenommen wor-

\footnotetext{
${ }^{31}$ Im Urteil 41 wurde gleichfalls befunden, dass das in dem Fall der römische Grundsatz gilt, ohne das näher zu erläutern.

${ }^{32} \mathrm{Nr}$ 32. Appellationserkenntniß des Riga'schen Rathes vom 18. Aug. 1867, No. 7474, in: ZWINGMANN, Civilrechtliche 63-67.

${ }^{33}$ Nr. 32. Appellationserkenntniß des Riga'schen Rathes vom 18. Aug. 1867, No. 7474, in: ZWINGMANN, Civilrechtliche 66.
}

den sind - egal für welche Zwecke - benutzen ohne dass gegen uns die Klage wegen der Grabstörung gestellt werden könnte. Von der Unterscheidung zwischen Privatfluss und anderen Flüssen ist hier keine Rede. ${ }^{34}$ In sich ist der Verweis auf die Digestenstelle auch nicht nötig, sondern sollte offenbar das Wissen des Richters zeigen.

\subsection{Geltung des römischen Rechts bejaht ohne Quellenverweise}

In ein paar Fällen (Urteile 44 und 48) wird entweder explizit oder implizit die Geltung der römischrechtlichen Prinzipien bejaht, ohne aber auf nähere Grundlagen in Quellen hinzuweisen. So z.B. in der folgenden Entscheidung über Hypotheken: „[...] das Prov. Recht [hat] das Princip der Spezialität nicht consequent durchgeführt, sondern im Wesentlichen die Regeln des römischen Pfandrechts noch aufrecht erhalten [...]“.35

\section{Fazit}

Welche Rolle die Quellenverweise im LECP für die Praxis hatten, kann man erst aus der Praxis selbst sehen. Auch aufgrund dieser begrenzten Untersuchung kann man schon behaupten, dass die Quellenverweise im LECP in die Argumentation der Gerichte einbezogen wurden. So hatte Ylander Recht, der die Quellentexte als Auslegungshilfe angesehen hat. Der Gesetzestext hatte aber in diesen Entscheidungen immer die primäre Bedeutung und ging dem Quellentext vor. Wohl haben sich die Gerichte nicht immer ,an die in den Fußnoten angegebenen Quel-

\footnotetext{
${ }^{34}$ Es stammt auch nicht von den unter den betreffenden Artikeln genannten römisch-rechtlichen Stellen (D 22, 1, 25, 1; Nov. 8, 3, 3; Glossa zu D 6, 3, 1.; D. 43.18 .3 (existiert nicht); C 10, 15,1); diese Texte behandeln eine andere Problematik.

${ }^{35}$ Nr. 48. Rechtskräftiges Locationsurteil der I. Section des Landvogteigerichts vom 17. Aug. 1868, No. 193, in: ZWINGMANN, Civilrechtliche 99.
} 
len“36 gehalten (Urteil 32) - obwohl sie sich nach Ylander daran halten hätten müssen. Rechtstheoretisch konnte die Meinung von Erdmann, dass die Kodifikation der Quellenstelle keine "gesetzesgleiche Charakter geben" wollte, richtig sein, doch die Praxis ist davon nicht ausgegangen. Obwohl der Gesetzestext die primäre Bedeutung hatte, wurden auch die Quellenverweise als Teil des Gesetzestextes - wenn auch mit sekundärer Bedeutung - angesehen.

Es muss gesagt werden, dass im Titel dieses Beitrags getroffene Gegenüberstellung von Legitimation und Rechtsauslegung nicht ganz richtig ist. Anscheinend können beide Ziele gleichzeitig figurieren. Dass ein Ziel der römischrechtlichen Quellen im LECP die Legitimierung des Gesetzbuches und der richtigen Quellen war, ist aufgrund bisheriger Untersuchungen ziemlich sicher. Die Benutzung der Quellenverweise zur Auslegung stand vielleicht nicht im Plan des Verfassers des LECP, Bunge, aber die ostseeprovinzielle Praxis hat die angebotene Möglichkeit jedoch benutzt. Wenn man aufgrund der Untersuchungen von Senatsurteilen sagen konnte, dass es eher Ausnahme als Regel war, ist es bei den Entscheidungen der Riga'schen Stadtgerichten anders. Die römischrechtlichen Quellen werden in 1/3 der Entscheidungen zitiert. Meistens sind sie nicht Teil der Hauptargumentation, aber auch nicht bloß "Verzierung", sondern wirklich eine Auslegungshilfe.

Es bestätigt sich die Behauptung, dass die ostseeprovinziellen Juristen gewohnt waren, die römischen Quellen zu benutzen. Daher war die Legitimierung des neuen Gesetzbuches durch die Quellenverweise gar kein schlechter Gedanke. Darin folgten die Richter derselben Linie wie die ostseeprovinziellen juristischen Schriftsteller, welche die Provinzialwissenschaft wieder mit der zeitgenössischen gemeinrechtlich-deutschen

\footnotetext{
${ }^{36}$ Ylander, Die Rolle 445. Siehe Punkt 2. des Aufsatzes.
}

Rechtswissenschaft verbinden wollten. ${ }^{37}$ Andererseits bedeuten auch richtige Quellenverweise nicht, dass der Richter sie zitiert oder gar richtig zitiert. Ein Richter hat seine Entscheidungsfreiheit mit oder ohne Quellenverweise, obwohl die letzteren einfacher $\mathrm{zu}$ benutzen sind, wenn sie Teil des Gesetzes sind.

Die Untersuchung bestätigt aber auch die vielleicht banal klingende, jedoch sehr richtige Ansicht, dass das Ausgehen nur von den Gesetzen (und deren Quellenanalyse) nicht die ganze juristische Wirklichkeit zeigt. Um dies zu sehen, muss auch die entsprechende Praxis untersucht werden.

\section{Korrespondenz:}

Hesi Siimets-Gross

Faculty of Law

Näituse 20, 50409 Tartu, Estonia

hesi@ut.ee
${ }^{37}$ LUTS, Juristischen Zeitschriften, 108-109. 


\section{Abkürzungen:}

LECP Liv-, Esth- und Curlaendisches Privatrecht

Nov. Novellae

\section{Literatur:}

Jörg BABEROWSKI,. Das Justizwesen im späten Zarenreich 1864-1914. Zum Problem von Rechtsstaatlichkeit, politischer Justiz und Rückständigkeit in Rußland, in: ZNR 13 (1991) 156-172.

Pio CARONI, Gesetz und Gesetzbuch. Beiträge zu einer Kodifikationsgeschichte (Basel-Genf-München 2003).

Carl E. ERdmann, System des Privatrechts der Ostseeprovinzen Liv-, Est- und Kurland, Bd. 1: Allgemeiner Theil. Familienrecht (Riga 1889).

Wilhelm GreIfFEnHagen, Dr. Jur. Friedrich Georg v. Bunge (Reval 1891).

Marju LUTS, Modernisierung und deren Hemmnisse in den Ostseeprovinzen Est-, Liv- und Kurland im 19. Jahrhundert, in: Tomas GIARO (Hg.), Modernisierung durch Transfer im 19. und frühen 20. Jahrhundert (Frankfurt am Main 2006).

Marju LUTS-SOOTAK, Das Baltische Privatrecht von 1864/65 - Triumphbogen oder Grabmal für das römische Recht im Baltikum?, in: Zeitschrift für Ostmitteleuropa-Forschung 58 (2009) 357-379.

Marju LUTS-SOOTAK, Die baltischen Privatrechte in den Händen der russischen Reichsjustiz, in: Zoran POKROVAC (Hg.), Rechtsprechung in Osteuropa. Studien zum 19. und frühen 20. Jahrhundert (Frankfurt am Main 2012).

Marju LUTS, Die juristischen Zeitschriften der baltischen Ostseeprovinzen Russlands im 19. Jahrhundert: Medien der Verwissenschaftlichung der lokalen deutschen Partikularrechte, in: Michael StOlleIS, Thomas SIMON (Hgg.). Juristische Zeitschriften in Europa (Frankfurt am Main 2006) $67-116$.
Marju LUTS, Private Law of the Baltic Provinces as a Patriotic Act, in: Juridica International 5 (2000) 157-167.

Carl Otto von MADAI, [Rezension von] F. G. Bunge, Das Liv- und Esthländisches Privatrecht, in: Kritische Jahrbücher für deutsche Rechtswissenschaft 5 (1841).

Provincialrecht der Ostseegouvernements. Dritter Theil. Privatrecht. Liv-. Est- und Curlaendisches Privatrecht. Zusammengestellt auf Befehl des Herrn und Kaisers Alexander II. (St. Petersburg 1864).

Hesi SIIMETS-Gross, Das Liv-, Esth- und Curlaendisches Privatrecht (1864/1865) - die einzige Quelle des Privatrechts?, in: Marju LUTS-SOOTAK, Sanita OsIPOVA, Frank L. SCHÄFER (Hgg.), Einheit und Vielfalt in der Rechtschichte im Ostseeraum/ Unity and Plurality in the Legal History of the Baltic Sea Area (Frankfurt am Main 2012).

Hesi SIIMETS-GROSS, Das „Liv-, Esth- und Curlaendisches Privatrecht" (1864/1865) und das römische Recht im Baltikum (=Dissertationes iuridicae Universitatis Tartuensis 33, Tartu 2011).

Hesi SiIMETS-Gross, Roman Law Origin of the Private Law Act of the German Baltic Provinces of the Russian Empire: Fact or Fiction?, in: Laura BECK VAlera, Pablo Gutiérrez VegA, Alberto SpinosA (Hgg.). Crossing Legal Cultures. (= Jahrbuch Junge Rechtsgeschichte - Yearbook of Young Legal History 2007, München 2009) 313-330.

Hesi SiImets-Gross, Roman Law in the Baltic Private Law Act - the Triumph of Roman Law in the Baltic Sea Provinces?, in: Juridica International 12 (2007) 146-155.

[http://www.juridicainternational.eu/public/pdf/ji _2007_1_180.pdf] (abgerufen am 12. 1. 2013].

Victor ZWINGMANN, Civilrechtliche Entscheidungen der Riga'schen Stadtgerichte, Bd. 1 (Riga 1871).

Aage Ylander, Die Rolle des römischen Rechts im Privatrecht der Ostseeprovinzen Liv-, Est- und Kurland, in: Zeitschrift für vergleichende Rechtswissenschaft 35 (1918). 